\title{
Improving DTR assessment by means of PCA applied to wind data
}

\author{
Alessandro Bosisio ${ }^{\mathrm{a}, *}$, Alberto Berizzi ${ }^{\mathrm{a}}$, Dinh-Duong Le ${ }^{\mathrm{b}}$, Fabio Bassi ${ }^{\mathrm{c}}$, Giorgio \\ Giannuzzi ${ }^{\mathrm{c}}$ \\ ${ }^{a}$ Politecnico di Milano, Department of Energy, Via la Masa 34, 20156 Milano, Italy \\ ${ }^{b}$ Faculty of Electrical Engineering, The University of Danang-University of Science and \\ Technology, Danang, Vietnam \\ ${ }^{c}$ TERNA S.p.a., Via dei della Robbia 41, 50132 Firenze, Italy
}

\begin{abstract}
Traditionally, the rating of an overhead transmission line is determined under a set of specified and standardized conditions. However, weather conditions along the line change during operation. Therefore, the standard rating of the line might be either underestimated, leading to inefficient utilization of the line, or overestimated, leading to unsecure operation. This is the major drawback of the traditional approach: the so-called Dynamic Thermal Rating (DTR), that takes into account the actual operating conditions along the line to determine the rating, is today a critical need. In this paper, we develop a comprehensive methodology for exploring all necessary information about stochastic processes of environmental variables surrounding and along the line using available data. The results can be used as input to determine the actual rating of the considered transmission line to enhance the determination of the rating for transmission lines.
\end{abstract}

Keywords: Dynamic Thermal Rating; Principal Component Analysis;

Forecast technique; Transmission line; Power system management

\footnotetext{
* Corresponding author

Email address: alessandro.bosisio@polimi.it (Alessandro Bosisio )
} 


\section{Introduction and motivation}

The rating of an overhead transmission line is the maximum current that the line is allowed to carry continuously without exceeding the maximum temperature of the conductor. Thus, temperature affects sags between towers and

5 tensile strength; it depends on the magnitude of current through the line, its time duration, and on environmental conditions, such as wind speed, air temperature, solar radiation, and so on [1, 2].

Traditionally, the rating is determined under a set of standard conditions [1, 2, 3]. However, the actual operating conditions, especially weather condi10 tions along the line, vary during operation. Therefore, the rating of the line might be either underestimated, leading to inefficient utilization of the line, or overestimated, leading to unsecure operation. This is why the so-called Dynamic Thermal Rating (DTR), that takes into account the actual operating conditions to determine the rating, is considered today a critical need.

15 DTR bring us several benefits 4. It allows the use of existing transmission assets efficiently, thus improving economic benefits, allows deferral of new transmission lines, it makes it possible to manage more easily contingency conditions. The better exploitation of available infrastructure is very useful in the presence of non programmable Renewable Energy Sources (typically, wind power), that often cause congestions in the system and wind power curtailment. DTR has been much cheaper than storage in solving this kind of problems in Italy. That is why, in the present paper, DTR is considered with reference to windy areas, where it can be adopted most profitably.

In order to assess the dynamic rating of the line and to schedule for the best operation for the next 5-15 minutes, necessary information could be provided by Weather Forecast Providers (WFPs); however, the forecast required for an efficient power system operation are not available from WFPs, especially for mountainous areas, where conditions might change very significantly and quickly and for each span of the transmission line. For this reason, Terna investment so plans include the installation of several sensors along many electrical lines to 
integrate information coming from WFPs with data coming in real-time by its own sensors.

Environmental variables such as wind, temperature, solar radiation, and so on can be considered as random variables that cover a wide geographic area 35 and evolve over time, determining the stochastic process. Hence, in order to obtain future information about it, a suitable space-time modeling method which can explore and capture all necessary information in the processes needs to be developed.

Future information on stochastic processes can be provided by forecast techniques such as point forecast and probabilistic forecast [5]. However, such techniques do not capture temporal correlation between forecast errors (i.e., uncertainty) of different time-steps. In a different way, scenario forecasts [6] can capture such information, especially temporal correlation, and give forecasting results in the form of a set of discrete scenarios. So far, several techniques

45 have been used for scenario generation. Reference [7] discusses on using various techniques including conditional sampling, property-matching methods, optimal discretization, and step-wise growing and cutting methods for generating scenarios for stochastic programming.

Scenario forecasts have been applied to several research areas in practice. In ${ }_{50}[8]$, the authors generate arrival capacity scenarios for each airport in United States based on non-parametric methods (in [9]) using only historical data. Clustering techniques are also used in that study, so that the scenarios generated are the averages of clusters of similar profiles.

The technique for scenario construction using both historical data and forecasted data is discussed in [10]: it shows a better performance compared to [8. In another field, reference [11] uses the daily pattern of change from Global Climate Models to generate rainfall scenarios. However, those methods are only suitable for generating scenarios for a single stochastic process (e.g., wind speed process at a single location).

In order to produce scenario forecasts for multiple stochastic processes which are cross-correlated, e.g., wind speed processes at multiple locations, space-time 
modeling techniques are needed including non-stationarity, non-Gaussianity, temporal and spatial correlation.

A careful analysis on the existing methods in this field is provided in [12, where the Authors use orthogonal Markov chain; however, in the paper the Authors assume spatio-temporal covariance stationarity, i.e., that the autocovariance function of each time series at each location and the spatial correlations among time series of different locations do not change.

In the field of wind power studies for generating space-time wind scenarios, in order to characterize interdependence structure of multivariate stochastic processes, Gaussian copula method [13] is widely used. In [14, the Authors build the model for multi-site wind speed using a noise vector deriving a vector auto-regressive process. This model accounts for both spatial and temporal correlations of wind speed and assumes joint Gaussian distribution and station-

75 arity. In [14] and [15], the problem is simplified by the assumption that the matrix of auto-regressive coefficients is diagonal, which implies that the multivariate time series model can be decoupled into different univariate time series model for each wind site.

This paper presents a new approach based on a space-time model that, using both weather historical data and real-time measurement data with different temporal resolutions, provides good forecasting for DTR of transmission lines. Specifically, the space-time model uses the analysis of current wind situation provided by a WPF (available every 12 hours with space resolution of $2 \mathrm{~km} \mathrm{x}$ $3 \mathrm{~km}$ ) integrated with real-time measurements available every 5 minutes at a significant number of different specific locations.

The paper is organised as follows: Section 2 motivates the proposed methodology. In Section 3 the Space-Time Model is presented, while Section 4 describes the real-time operation of the proposed procedure. Section 5 reports the results of the procedure developed, comparing wind forecasting data and real measure90 ment data to check the accuracy of the methodology. Concluding remarks are given in Section 6 


\section{Integration of weather data for the DTR of transmission line}

In Italy, windy areas show a high penetration of wind power generation that, in windy conditions, are currently limited by the presence of transmission congestions. However, depending on the wind speed and direction, wind can itself contribute to increase the actual rating of the line, thus alleviating congestions. The goal of the present paper is to estimate the wind speed (magnitude and direction) along the spans of an electrical line: the estimated values are then transferred to a line rating model in order to complete the DTR of the line and possibly avoid wind generation curtailment.

The proposed approach aims at integrating the available information sources, which are very different in terms of time and space resolution:

- Weather Forecast Provider (WFP) information: they are high level weather data analysis based on a mathematical-physical model computed according to a very refined spatial mesh for the whole continent. Data are available every 12 hours (at 00:00 and 12:00 every day) for many layers in height;

- Real-time (RT) weather stations data: the Italian TSO, Terna, has been installing for some years many sensors that can measure weather data (temperature, wind, etc.) and transfer such information to the control centre. Typically, such devices are installed on top of quite a few towers along some transmission lines (TL). They provide real-time measurements at their location only at a 5 minute rate; of course, they are scattered on the territory, not on a regular basis, unlike WFP information.

The problem is how to integrate such two set of data: WFP data, given for a very large area with a refined mesh, available with a very poor time resolution, with the RT measurements immediately available for scattered locations along some electrical lines with very good time resolution. The goal is to make it possible to determine reliably the wind conditions along a chosen electrical line for the next 5 minutes to 2 hours, in order to make it possible to keep the rating 
of the lines updated and redispatch in real-time, if possible, some power that should be curtailed if the DTR were not available.

\subsection{WFP data}

WFP data are supposed to provide a general picture of how the wind streams are moving in a large scale. As shown in Fig. 1a, where an area in the CentreSouth of Italy is shown, weather data are provided for a selected rectangular area with a resolution of $2 \mathrm{~km}$ for the longitude and $3 \mathrm{~km}$ for the latitude. Data on all the points of the mesh, marked with yellow triangles, are computed by the WFP in two snapshots are at 00:00 and 12:00 UTC. Hence, WFP data have a high spatial resolution but a poor temporal resolution, and are related to a mathematical-physical model, not to measured data. Therefore, they are useful to extract a time-space model, but not to be used immediately for the DTR. In the proposed approach, the WFP data are used to create and characterize a database of past long-term scenarios, to be used to predict future long-term scenarios. A scenario derived by WFP data includes weather features at every point in the mesh, with time resolution equal to 12 hours. It should be considered as useful to learn weather behavior on a larger-scale area. Such characteristics will be integrated with data coming from RT stations.

As it is clear, WFP dataset is very huge. In order to reduce its size, the following criteria are adopted:

- for WFP mesh points surrounding the considered TL, the highest available resolution $(3 \mathrm{~km} \times 2 \mathrm{~km})$ is kept;

- for WFP mesh points far from the transmission line, the resolution is decreased $(6 \mathrm{~km} \times 4 \mathrm{~km})$;

- at any rate, the four closest WFP mesh points around each RT stations are always kept, independent of their distance from the TL, with the goal of identifying and exploiting as much as possible the correlation between WFP and RT data. 
In Fig. 1a areas with a higher space resolution can be observed around both RTs and the considered TL.

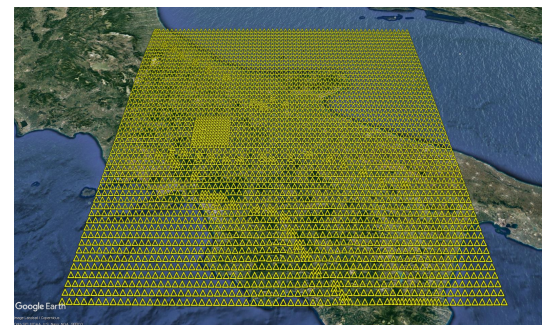

(a) WFP mesh on Southern Italy

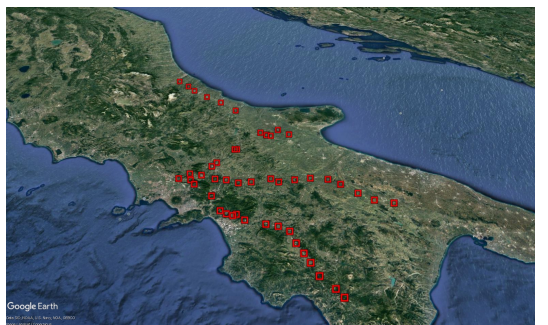

(b) RT stations

Figure 1: WFP mesh and RT station locations

The vector of wind speed data can be expressed in terms of orthogonal speed components, where:

- $u$ is the Zonal speed, the component of the horizontal wind towards East;

- $v$ is the Meridional speed, the component of the horizontal wind towards North.

Wind data are given for each point of the mesh and for 6 different heights above the ground (layers $10 \mathrm{~m}, 30 \mathrm{~m}, 50 \mathrm{~m}, 70 \mathrm{~m}, 100 \mathrm{~m}$ ). Based of the specific TL architecture, suitable height is considered.

WFP data are given by the WFP in GRIB format (GRIdded Binary) a data format standardized by the World Meteorological Organization's Commission.

\subsection{Real-time data}

Real-time (RT) measurements recording is carried out, with a temporal resolution of 5 minutes, at 43 weather stations installed on a TL tower or in a substation. Geographical locations of the RT measurement stations considered in this paper, marked with red squares, are shown in Fig. 1b.

RT data including, for each sensor, time-stamped wind magnitude and direction, are transferred every 5 minutes to the TSO control centre. Like any real-time data flow, RT data can be affected by missing or erroneous values 
in the RT dataset. This issue must be taken into account in the time-space modelling.

\subsection{TL considered for DTR}

In order to select data for the time-space model, it is necessary to define the TL subject to DTR. Fig. 2 shows the TL located in the Southern Italy considered for DTR with its 124 towers. This TL is also equipped with 4 RT stations marked with red squares in Fig. 2. TL has average height above ground of $25 \mathrm{~m}$ (height of the lowest conductor and sensors, when installed).

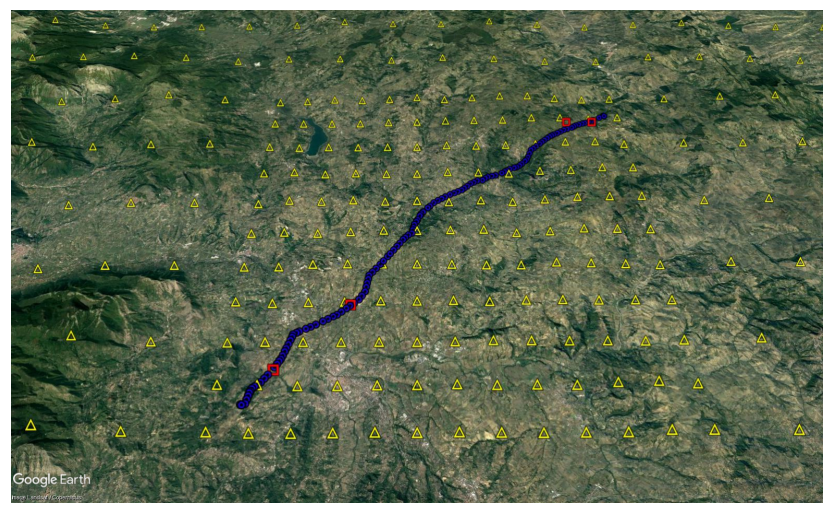

Figure 2: TL path, in the WFP mesh

\section{Space-Time Model}

The core of the forecasting approach to enhance DTR assessment is an extension of the space-time modeling presented in [16. It combines Principal 180 Component Analysis (PCA) with clustering/selection, extrapolation, interpolation techniques. It can capture all salient features of wind speed from multiple locations: non-Gaussianity, non-stationarity with temporal and spatial correlations thanks to PCA, which also provides an excellent tool for approximating a large data set by reducing its size. 
PCA is an orthogonal transformation of a generic data set into an set of uncorrelated data [17, 18. It is based on the eigenanalysis of the covariance matrix (or the correlation matrix) of a set of data, that determines a transformation matrix such that transformed data $\mathbf{Z}$ show diagonal covariance matrix. The new variables are called Principal Components (PCs) and the technique is used to reduce as much as possible redundancy in the data set, while keeping only the first PCs.

Wind data are stored in a matrix $\mathbf{W}$ whose elements $w_{s}^{h}$ are the wind speed at site $s \in\{1,2, \ldots, S\}$ and at time $h \in\{1,2, \ldots, N\}$. We assume that all considered wind sites have the same number of observations $N$ and that they are synchronized and equally spaced in time.

$$
\mathbf{W}=\left[\begin{array}{cccc}
w_{1}^{1} & w_{1}^{2} & \cdots & w_{1}^{N} \\
w_{2}^{1} & w_{2}^{2} & \cdots & w_{2}^{N} \\
\vdots & \vdots & \ddots & \vdots \\
w_{S}^{1} & w_{S}^{2} & \cdots & w_{S}^{N}
\end{array}\right]
$$

At first, data are centered [17] in matrix $\boldsymbol{W}_{\boldsymbol{c}}$ by subtracting the mean $\mu_{s}$ of each time series at each site. In the following, the covariance matrix $\boldsymbol{\Sigma}$ is considered, for the sake of simplicity [17, whose elements are, in the diagonal, $i$ and the time series at site $j \sigma_{i, j}^{2}$.

Its eigenvalues $\lambda_{i}, i=1,2, \ldots, S$, real positive, and the associated eigenvectors $\mathbf{u}_{i}$ are considered and form the matrix $\mathbf{U}$ :

$$
\mathbf{U}=\left[\begin{array}{lllllll}
\mathbf{u}_{1} & \mid & \mathbf{u}_{2} & \mid & \cdots & \mid & \mathbf{u}_{S}
\end{array}\right]
$$

Its elements are also known as PC coefficients or loadings. Finally, PCs are 205 derived [17] as

$$
\mathbf{Z}=\mathbf{U}^{T} \boldsymbol{W}_{\boldsymbol{c}}
$$


where $\mathbf{Z}$ is a $S \times N$ matrix. The $i$-th row of matrix $\mathbf{Z}, \mathbf{z}_{i}$, is the $i$-th $\mathrm{PC}$, that is, a time series univariate and uncorrelated with other PCs [19].

The reconstruction of wind data from PCs is implemented inversely:

$$
\begin{aligned}
\mathbf{W}=\boldsymbol{\mu}+\mathbf{U Z}=\boldsymbol{\mu}+ & {\left[\begin{array}{lllllll}
\mathbf{u}_{1} & \mid & \mathbf{u}_{2} & \mid & \cdots & \mid & \mathbf{u}_{S}
\end{array}\right] } \\
& \cdot\left[\begin{array}{lllllll}
\mathbf{z}_{1}^{T} & \mid & \mathbf{z}_{2}^{T} & \mid & \cdots & \mathbf{z}_{S}^{T}
\end{array}\right]^{T}
\end{aligned}
$$

It is worth noting that if the distribution considered is multivariate Gaussian, resulting PCs will be independent. Otherwise, PCs will be uncorrelated but still dependent (the diagonal covariance matrix of PCs only implies that they are uncorrelated). In the present paper, we adopt pre-processing and transformation techniques to obtain approximately stationary and Gaussian data sets to improve PCA.

PCA is an excellent tool to approximate a large data set by reducing its dimension [18. This function makes PCA a powerful tool for high-dimensional data analysis. Each $\mathrm{PC} \mathbf{z}_{l}$ holds an amount of information which is represented by its variance, i.e., by $\lambda_{l}$. Therefore, the contribution of the $l$-th PC to total variance of the data [18] can be computed as:

$$
\gamma_{l}=\frac{\lambda_{l}}{\sum_{i=1}^{S} \lambda_{i}} \times 100 \%
$$

Hence, the first row vector $\mathbf{z}_{1}$, corresponding to the largest eigenvalue $\lambda_{1}$, is 220 the most dominant component, which contains most of the variance in the data set, followed by the second component $\mathbf{z}_{2}$, and so on. This makes it possible to approximate the initial data set by a few PCs, based on the total amount of information. This makes it possible to describe most of the features of the data set by a reduced number of variables. PCA is used in this paper to reduce 225 the number of variables without losing important information and to carry out time series analysis and scenario generation on a few PCs instead of trying to do the same working on longer data sets. 


\subsection{Time series analysis}

A time series is a sequence of observations ordered in time, usually at equally-

It should be noted that stationarity is a necessary condition in building an ARMA model. However, this condition may not always hold with real time series data. In such a case, data must be pre-processed. In this paper, we carry out various pre-processing and transformation techniques. At first, input data 240 (usually non-stationary and non-Gaussian) are pre-processed and transformed to obtain stationary and Gaussian data as required. The input for space-time model is matrix $\mathbf{W}$; all time series must span the same time horizon with equal intervals (12 hours for WFP mesh and 5 minutes for RT measurements). After that, PCA is carried out and a few (depending on their variance) PCs are fitted by a time series model (to include variability content), and used to generate an adequate number of time series for future time. The generated time series in terms of PCs are back transformed into non-Gaussian data [14, 15] to obtain scenarios obeying all the characteristics of the observed wind data for each site.

Hence, the space-time modeling method can capture most of the main characteristics of the input data and gives the output results in terms of scenarios of future values (with a certain future time frame, e.g., 12 hours ahead) for each input quantity with the same temporal resolution as the input data. The novelty of the proposed approach is that it can explicitly capture the main features of stochastic processes of multi-site wind data: marginal distribution, spatial correlation, temporal correlation, diurnal and seasonal non-stationarity and non-Gaussianity.

It is also worth noting that PCA allows a significant reduction of the size of the input data set without losing significant information. This feature is very 
useful because the size of data set used in this research is very large, due to a

\section{The proposed methodology}

As mentioned, the most challenging issue faced is the integration of data coming from different sources, to identify the most probable values of wind speed along the TL to be used for the DTR. In this Section, we present a comprehensive framework proposed for exploiting available wind data along a TL to provide useful information for improving DTR assessment. The goal is to get, for each point along the electrical line, reliable forecast of the wind (speed 
and direction) for the next couple of hours with a time resolution of 5 minutes. This is obtained by generating a number of scenarios with different space and time resolution making use of the PCA approach. The whole structure of the procedure implementation is depicted in Fig. 3.

Two main databases have to be created, with different time resolution:

- DB1 contains wind data with 12 hours time resolution, coming from both WFP model, relevant to each point considered in the mesh, and additional data synchronized in time with WSP data (i.e., every 12 hours), coming from all RT sensors available. PCA working on data from DB1 generates 12-hour resolution scenarios.

- DB2 contains data coming every 5 minute from the RT measurements. They are used to generate scenarios with 5-minute time resolution at RT locations using PCA.

Both databases are created initially by InputWFP an InputRT and updated by blocks Update WFP and UpdateRT as soon as new data are available, i.e., either every 12 hours or every 5 minutes, respectively. Actually, the same procedure is carried out for two components, namely $\mathrm{u}$ and $\mathrm{v}$, of wind separately.

The generation of 12-hour scenarios is carried out within Block1, every 12 hours, when new analysis data from the WFP are available in DB1. It includes the Space-Time Modeling (STM1) presented in Section 3 which aims at capturing all main features of the wind stochastic processes at points on both the WFP mesh and the RT stations and then at generating scenario forecasts at all these points, but in particular around the TL under DTR, over a predefined future time frame (for example 12 or 24 hours) and with time resolution of 12 hours. In Block1, due to high computation times that would be needed by the PPCA (PSTM1), the choice has been made for an efficient technique for missing data and outliers treatment combined with PCA. Moreover, since WFP data come from a model and it is assumed to be reliable, this issue is less significant than for the RT data treatment. N1 scenarios are generated by the space-time model and stored in database SDB1. 


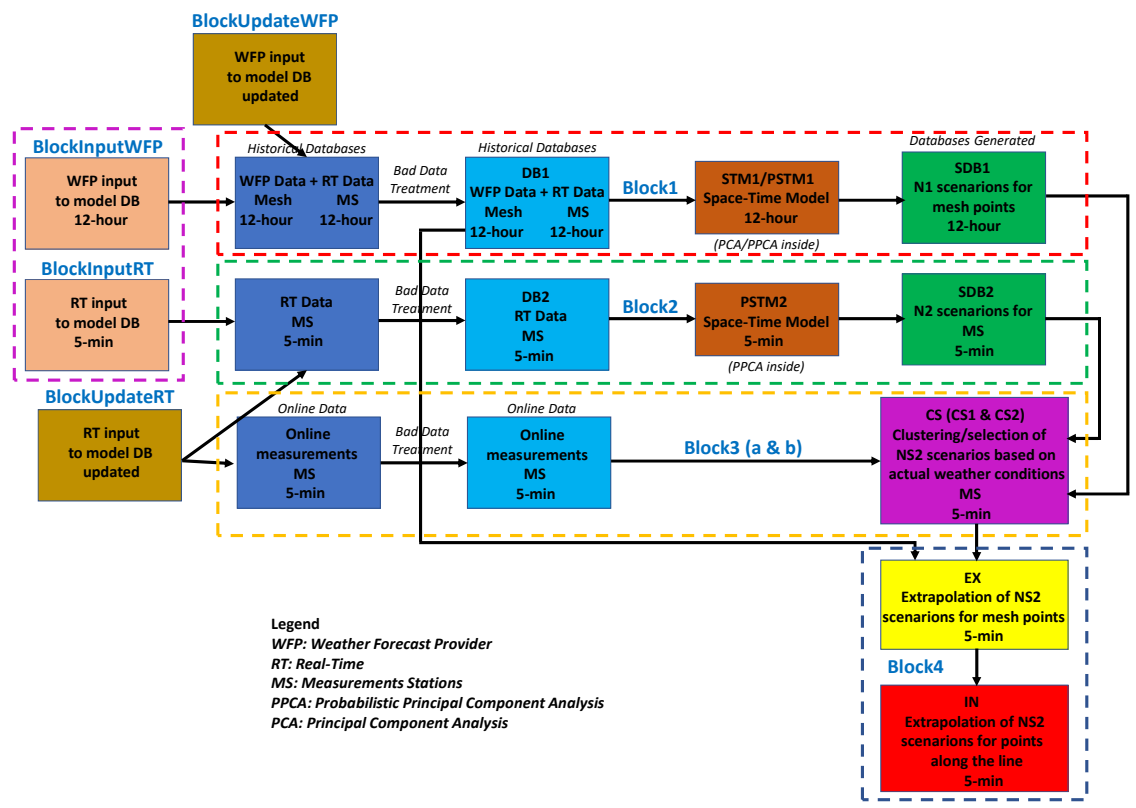

Figure 3: Block diagram of the proposed methodology

In the same way, in Block2, the same is done by STM2 for RT data in DB2, in order to generate scenarios to be stored in SDB2. Data are those relevant to RT measurement locations, with 5-minute time resolution. This block is run every hour, when a set a new data from the RT measurements is newly available and generates N2 scenarios over a predefined future time frame of 2 hours with time resolution of 5 minutes. Since data come from real-time measurements, thus being affected by missing values and outliers, and since the RT stations are fewer than mesh nodes, the procedure uses PPCA (PSTM2) to deal with missing or bad data.

The goal of Block3 is to pick up a few scenarios closest to the actual weather conditions. This is done by a clustering/selection procedure (i.e., block $C S$ in Fig. 3). Block3 is in turn divided into two blocks: Block3a, which works with $S D B 1$ and, using CS1, selects NS1 scenarios among N1 12-hour scenarios (it 
is run every 12 hours), and Block3b, which works with SDB2 and using CS2 selects NS2 scenarios among N2 5-minute scenarios (it is run every hour). In fact, during the real-time operation, online measurement data at RT stations (online data in Fig. 3) are collected. These data are combined with SDB1 and SDB2 to select NS2 most probable scenarios (5-min resolution) for each quantity at RT stations.

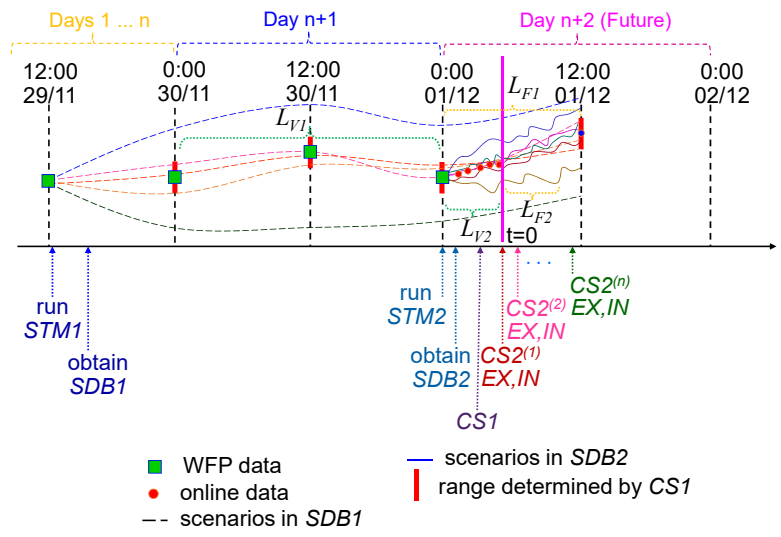

Figure 4: Example of choosing suitable time instances to start running each block

For the sake of clarity, Fig. 4 describes an example of choosing suitable time instances to start running each block. The schedule is made based on both the running time needed to perform each block and the availability of updated input data. Focusing on block $C S$ of Fig. 3. two stages of selection (corresponding to two sub-blocks $C S 1$ and CS2) are needed:

- CS1: selection of NS1. In the example of Fig. 4, STM1 uses data collected up to 29/11 to generate $N 1$ scenarios for four steps ahead (i.e., 0:00 and 12:00 on $30 / 11,0: 00$ and 12:00 on 01/12). With CS1, the procedure selects NS1 scenarios whose paths are most similar to WFP data at 0:00 and $12: 00$ on $30 / 11$ and $0: 00$ on 01/12 (i.e., latest known data used as validation set $-L_{V 1}=3$ ). Once $N S 1$ scenarios are identified, they also provide information on most likely range of values for each variable at 
12:00 01/12 (unknown/forecasted data, range in red in Fig. 4); $D_{w_{i}, y_{v}}$.

Similarly, as shown in Fig. 4 for $C S 1$ we considers $K=L_{V 1}=3$ (latest three set of data in $D B 1$ ).

At this point we have, for each RT station, a 5-minute resolution forecast

the same idea of CS1, CS2 selects NS2 scenarios which fulfill both following constraints: their paths are most similar to the latest and current RT measurements, and their values at 12:00 on 01/12 are within the range determined by CS1. Based on the need of forecast for the next 2 hours, CS2 is implemented in real-time: every hour, when a set of new twelve 5-minute data at RT measurement stations are available, CS2 is run to select the best NS2 scenarios in SDB2 for the next 2 hours (2 hours intervals correspond to $L_{F 2}=24$ time-steps of 5 minutes in the future). In Fig. 4, $L_{F 2}$ and $L_{V 2}$ are number of forecasted values and number of values in validation set. The process is repeated (called $\left.C S 2^{(2)}, \ldots\right)$ until the next PSTM2 is carried out.

To select the best NS2 scenarios (time series) among N2 scenarios in SDB2, the similarity between each time series $i$ in $N 2\left\{w_{i}^{k}\right\}$ and time series of the validation set $\left\{y_{v}^{k}\right\}$ is computed:

$$
D_{w_{i}, y_{v}}=\frac{1}{K} \sqrt{\sum_{k=1}^{K}\left(w_{i}^{k}-y_{v}^{k}\right)^{2}}
$$

where:

$\left\{y_{v}^{k}\right\}(k=1,2, \ldots, K)$ includes $L_{V 2}$ latest online measurement data and one point determined by $C S 1$ (i.e., $K=L_{V 2}+1$ );

$\left\{w_{i}^{k}\right\}(k=1,2, \ldots, K ; i=1,2, \ldots, N 2)$ is $i$ in SDB2 where $\left\{w_{i}^{k}\right\}$ is synchronized with $\left\{y_{v}^{k}\right\}$.

NS2 scenarios are selected, corresponding to the NS2 smallest values of

included in the NS2 scenarios selected. The last step is then to move to 5- 
minute resolution scenarios at points along the line; this is done in Block 4 which includes Extrapolation and Interpolation.

Extrapolation aims at computing, on the grounds of both the current RT measurements and NS1 and NS2 scenarios selected, 5-minute forecasted scenar-

\section{Computational experiments and results}

In this section, some selected results related to the space-time model applied to the TL shown in Fig. 2 are reported. As WFP analysis, we considered wind 


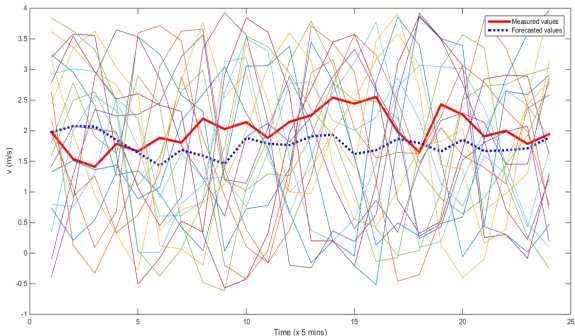

(a) Forecasting for RT station $4-\mathrm{u}$ component

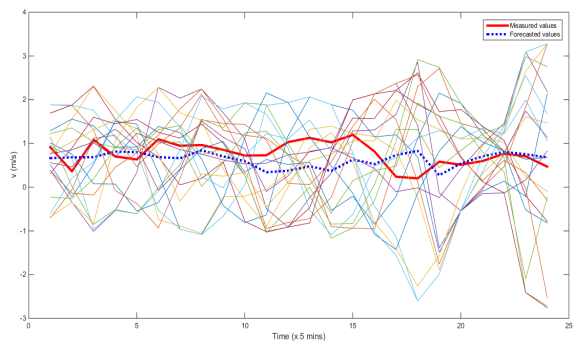

(b) Forecasting for RT station $4-\mathrm{v}$ component

Figure 5: Output of the forecasting process for RTs

To access quantitatively the capabilities of the method, the Average Root Mean Square error (ARMS) and Mean absolute error (MAE) are calculated, for all the RT stations, as the distance between actual and estimated data.

As shown in Fig. 6 and 7, the average ARMS errors is around $0.3 \mathrm{~m} / \mathrm{s}$ while the average MAE is around $1 \mathrm{~m} / \mathrm{s}$.

An example of the output of the methodology is shown in Fig. 8. It depicts the 20 best scenarios interpolated from the 4 closest WFP mesh points surrounding TL tower 1 and, again in dashed blue line, the mean values of those 


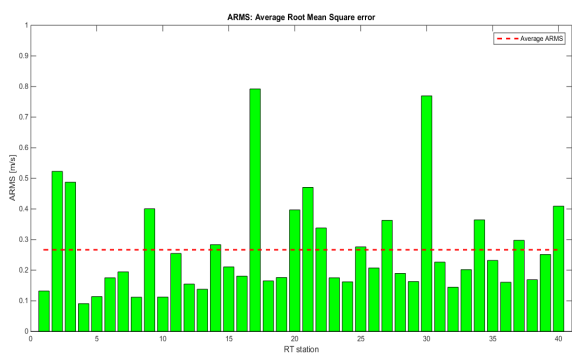

(a) ARMS - u component

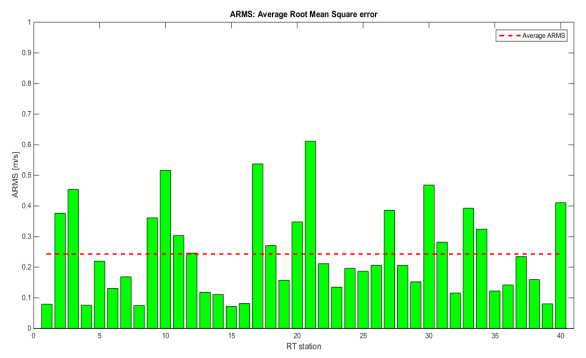

(b) ARMS - v component

Figure 6: RT stations ARMS

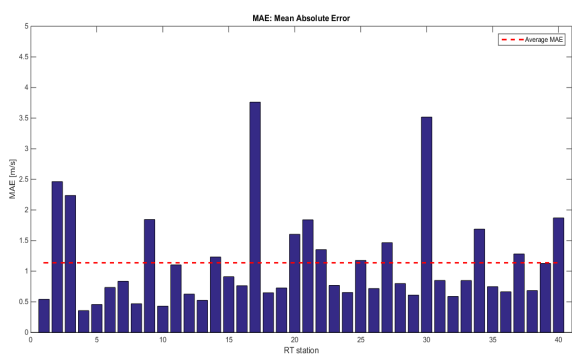

(a) MAE - u component

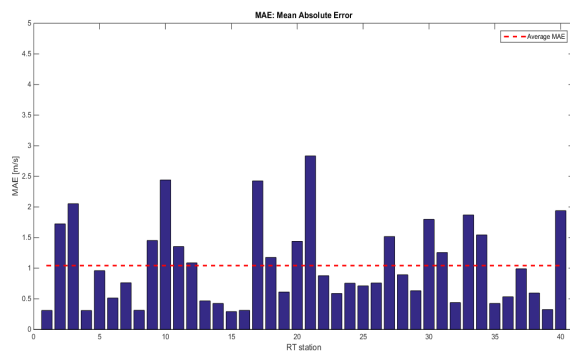

(b) MAE - v component

Figure 7: RT stations MAE

425

scenarios (which is considered as the wind forecast for TL tower 1). The accuracy of the wind forecasting at tower 1 cannot be assessed because no measured data are available at tower 1 . The methodology gives a similar wind forecasting for all the other $123 \mathrm{TL}$ towers as well as for any point of the TL.

\section{Concluding remarks}

430

In this paper, a methodology for exploring main characteristics of stochastic processes (e.g., non-Gaussianity, non-stationarity with distinct diurnal and seasonal patterns, temporal and spatial correlations) of wind speed along a TL is presented. All available data (both historical and RT data from a WFP and RT stations) are used to provide as much information about wind speed as possible 435 for a desired future time horizon for dynamic rating assessment. In particular, 


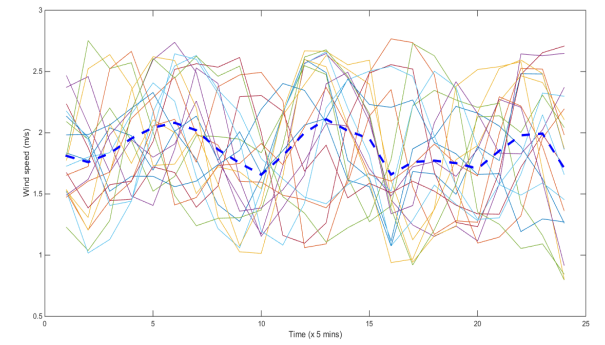

(a) Forecasting for the TL tower $1-\mathrm{u}$ component

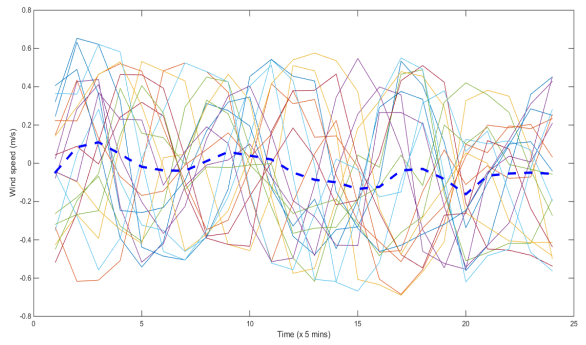

(b) Forecasting for the TL tower 1 - v component

Figure 8: Online forecast for TL towers

no simplifying assumptions are used for building the proposed model and the proposed procedure is very powerful for dealing with a high-dimensional data set due to a large number of variables associated with numerous points on the mesh.

For practically testing the proposed methodology, real data related to a TL in the province of Benevento (Italy), the WFP mesh surrounding it and RT stations included in the selected mesh have been considered. Simulation results are presented to show that the methodology proposed can improve the wind speed forecast and consequently the DTR of TL.

\section{References}

[1] IEC - International Electrotechnical Commission, Overhead electrical conductors - Calculation methods for stranded bare conductors, IEC, standard 61597 (1995).

[2] IEEE Standard, IEEE Standard for Calculating the Current-Temperature of Bare Overhead Conductors, IEEE, standard 738 (2006).

[3] S. D. Kim, M. M. Morcos, An application of dynamic thermal line rating control system to up-rate the ampacity of overhead transmission lines, IEEE Transactions on Power Delivery 28 (2) (Apr. 2013) 1231-1232. 
[4] F. Teng, R. Dupin, A. Michiorri, G. Kariniotakis, Y. Chen, G. Strbac, Understanding the benefits of dynamic line rating under multiple sources of uncertainty, IEEE Trans. Power Syst. 33 (3) (May 2018) 3306-3314.

[5] A. Botterud, Z. Zhou, J. Wang, R. J. Bessa, H. Keko, J. Mendes, J. Sumaili, V. Miranda, Use of wind power forecasting in operations decisions, Argonne National Laboratory, Technical report (Sep. 2011).

[6] P. Pinson, H. Madsen, H. A. Nielsen, G. Papaefthymiou, B. Klockl, From probabilistic forecaststo statistical scenarios of short-term wind power production, Wind Energy 12 (1) (2009) 51-62.

[7] M. Kaut, Scenario Generation for Stochastic Programming: A Practical Introduction, Tutorial on Scenario Generation, Håholmen (June 10-12, 2006).

[8] P. B. Liu, M. Hansen, A. Mukherjee, Scenario-based air traffic flow management: From theory to practice, Transportation Research Part B: Methodological 42 (7) (2008) 685-702.

[9] J. Dupacova, G. Consigli, S. W. Wallace, Scenarios for multistage stochastic programs, Annals of Operations Research 100 (1) (2000) 25-53.

${ }_{470}$ [10] G. Buxi, M. Hansen, Generating day-of-operation probabilistic capacity scenarios from weather forecasts, Transportation Research Part C: Emerging Technologies 33 (2013) 153-166.

[11] T. I. Harrold, R. N. Jones, Generation of rainfall scenarios using daily patterns of change from gcms, Water Resources Systems-Water Availability and Global Change (Proceedings of symposium IUGG2003), Sapporo, Japan (July 2003) 165-172.

[12] G. A. Baigorria, J. W. Jones, Gist: A stochastic model for generating spatially and temporally correlated daily rainfall data, Journal of Climate 23 (2010) 5990-6008. 
[13] J. Tastu, P. Pinson, H. Madsen, Space-time scenarios of wind power generation produced using a Gaussian copula with parametrized precision matrix, Technical University of Denmark, Technical report (2013).

[14] J. M. Morales, R. Minguez, A. J. Conejo, A methodology to generate statistically dependent wind speed scenarios, Applied Energy 87 (2010) 843-855.

[15] A. Papavasiliou, S. S. Oren, Stochastic modeling of multi-area wind power production, 12th International Conference on Probabilistic Methods Applied to Power Systems, Istanbul, Turkey.

[16] D. D. Le, G. Gross, A. Berizzi, Probabilistic modeling of multisite wind farm production for scenario-based applications, IEEE Trans. Sustainable Enery 6 (3) (Jul. 2015) 748-758.

[17] J. E. Jackson, A Users Guide to Principal Component Analysis, New York: Wiley, 1991.

[18] I. T. Jolliffe, Principal Component Analysis, New York: Springer, 2002.

[19] G. E. P. Box, G. M. Jenkins, Time Series Analysis: Forecasting and Control, San Francisco, CA: Holden-Day, 1976.

[20] M. E. Tipping, C. M. Bishop, Probabilistic principal component analysis, Journal of the Royal Statistical Society: Series B (Statistical Methodology) 61 (3) (1999) 611-622.

[21] F. Grubbs, Procedures for detecting outlying observations in samples, Technometrics 11 (1) (Feb. 1969) 1-21.

[22] IEC - International Electrotechnical Commission, Wind Turbines - Part 12-1: Power Performance Measurements of Electricity Producing Wind Turbines, IEC, iEC-61400-12 (2005).

[23] J. Li, A. D. Heap, A review of spatial interpolation methods for environmental scientists, Geoscience Australia, 2008. 